\title{
Fault Detection in Liquid-Propellant Rocket Engines Based on Improved PSO-BP Neural Network
}

\author{
Ningning $\mathrm{Li}^{1,2}$, Wei Xue ${ }^{2}$, Xiang $\mathrm{Guo}^{3}$, Liang $\mathrm{Xu}^{1 *}$, Yuyang $\mathrm{Wu}^{1}$, Yuan Yao ${ }^{1}$ \\ ${ }_{1}^{1}$ Tianjin Key Laboratory for Control Theory \& Applications in Complicated Systems, and School of Electrical \\ and Electronic Engineering, Tianjin University of Technology, Tianjin, 300384, China. \\ 2 Beijing Aerospace Propulsion Institute, Beijing 100076, China. \\ ${ }^{3}$ Big data Center, PICC Property and Casualty Company Limited, Beijing 100022, China. \\ * Corresponding author. Tel.: 13389951508; Email: liangx999@163.com \\ Manuscript submitted May 4, 2019; accepted July 10, 2019. \\ doi: 10.17706/jsw.14.8.380-387
}

\begin{abstract}
A method that uses an improved particle swarm optimization (PSO) algorithm combined with a backpropagation (BP) neural network is proposed to solve the problem of liquid-propellant rocket engine (LRE) failure detection. In the improved PSO algorithm, the global extremum is randomly perturbed by adding disturbance factor using the degree of particle aggregation around the global optimal value, and the individual extremum is randomly perturbed by adding disturbance factor using the number of particle extreme stagnation steps, disturbance factors are randomly added to individual extremum to diturb the particle's current search path, increasing the probability of particles jumping out of local extremum, avoiding the occurrence of local extremum, premature convergence or stagnation. In this paper, the improved algorithm is applied to the fault detection of a typical liquid rocket engine in steady state process. The simulation results show that, under the same conditions, the convergence speed of this PSO-BP method is obviously higher than that of BP neural network, and it does not fall into the local extreme value. The accuracy of fault detection is also improved significantly.
\end{abstract}

Key words: Liquid-propellant rocket engine, fault detection, particle swarm optimization (PSO), randomly perturbed.

\section{Introduction}

Liquid-propellant rocket engine (LRE) has many advantages, such as high performance, easy control and mature technology. It has been widely used in launch vehicle, ballistic rocket and spacecraft [1]. With the development of space, the launch quality of the carrier rocket is gradually increasing. According to statistics, in the United States, the failure of the power system accounts for more than $60 \%$ of the total failure of the launch vehicle [2]. Among the 54 space vehicle failures that occurred in the world from 1990 to 2002, there were 26 failures caused by the engine [3]. The demand for low-cost and high reliability has become even more important because of growing demand in the commercial launch market. Improving the reliability of LRE, in addition to strictly controlling the development process, should be based on component and machine reliability, monitoring and test verification, which rely heavily on health monitoring technology (HMT). Liquid-propellant rocket HMT mainly involves fault detection, fault diagnosis and fault control. Fault detection refers to data that are measured by a sensor and reflect the current working state of the engine. 
After feature extraction, binary detection is conducted, that is, whether an abnormality has occurred in the engine or if everything is normal. Fault isolation involves determining the location and type of fault based on existing abnormal status information, such as determining whether the pipeline is faulty, leaked or blocked. Fault identification is used to estimate the degree, size and properties of the fault.

In modern artificial intelligence fields, pattern recognition, neural networks and expert systems have all been applied to the detection and diagnosis of LRE faults. In particular, neural networks have good application prospects in LRE fault detection. A method for engine state monitoring combined with a BP network and an adaptive resonance theory network has also been proposed [4]. In addition, a fault diagnosis method using a turbopump parallel BP network has been studied [5]. A hybrid PSO algorithm has also been proposed to optimize a power system [6]. The current paper proposes to introduce particle aggregation degree into particle swarm, to judge the diversity of population, to prevent particles from gathering too much and to implement dispersal strategy on particle in time, so that the algorithm can avoid falling into local optimum. At the same time, the improved particle swarm optimization and BP neural network are combined to detect the failure of a typical liquid rocket engine during steady operation.

\section{PSO Algorithm}

The PSO proposed by Eberhart and Kennedy in 1995 is a global optimization evolutionary algorithm which is derived from the simulation of foraging behavior of birds and fish populations. It is similar to genetic algorithm, PSO is based on the concepts of population and fitness. A possible solution to the problem of individual representation in a particle swarm. Each particle has two descriptions of position and velocity. The objective function value corresponding to the particle position coordinates can be used as the fitness of the particle PSO algorithm to evaluate the particle fitness. It is different from genetic algorithm. PSO is not evolved by genetic operator, but by cooperation and competition among individuals to find the optimal solution. PSO has the advantages of fast convergence, simple operation and easy realization, without codec, selection, hybridization, mutation and other complex operations of genetic algorithm.

In the continuous space coordinate system, the mathematical description of PSO is as follows [7].

A group of $m$ particles flies at a certain speed in a D-dimensional search space. When each particle searches, it takes into account the historical best points it has searched for and the historical points of other particles within the group. The $i$ th particle has a particle group composed of three vectors:

Current position: $x_{i}=\left(x_{i 1}, x_{i 2}, \cdots, x_{i D}\right)$

Historical optimal position: $p_{i d}=\left(p_{i 1}, p_{i 2}, \cdots, p_{i D}\right)$

Velocity: $v_{i d}=\left(v_{i 1}, v_{i 2}, \cdots, v_{i D}\right)$

where $i=1,2,3, \cdots, m$. The current position is regarded as a set of coordinates describing the spatial point. In addition, the best position searched so far in the entire particle group is denoted as $p_{g d}=\left(p_{g 1}, p_{g 2}, \cdots, p_{g D}\right)$, which is generally called a global optimum. For each particle, its d dimension $(1 \leq$ $\mathrm{d} \leq \mathrm{D}$ ) changes according to the following equation:

$$
\begin{gathered}
v_{i d}(t+1)=v_{i d}(t)+c_{1} r_{1}\left(p_{i d}-x_{i d}\right)+c_{2} r_{2}\left(p_{g d}-x_{i d}\right) \\
x_{i d}(t+1)=x_{i d}(t)+v_{i d}(t+1)
\end{gathered}
$$

where $i=1,2,3, \cdots, m ; \quad r_{1}$ and $r_{2}$ are random numbers that follow a $\mathrm{U}(0,1)$ distribution, the learning factors $c_{1}$ and $c_{2}$ are non-negative constants, Usually, $c_{1}=c_{2}=2$, but other values can also be used $[7,8]$. To 
further improve the performance of the algorithm, Shi and Eberhart [9] increased the ability of particles to jump out of local extrema by adding a linear decreasing inertia weight (LDW) to equation (1). At the same time, $\omega$ determines the degree of influence of the previous velocity of the particle on the current velocity, thus playing a role $\omega$ in balancing the algorithm's global and local search abilities. The revised equation is as follows:

$$
v_{i d}(t+1)=\omega^{*} v_{i d}(t)+c_{1} r_{1}\left(p_{i d}-x_{i d}\right)+c_{2} r_{2}\left(p_{g d}-x_{i d}\right)
$$

The weight $\omega$ decreases linearly with the iterative running algebraic $t$ :

$$
\omega=\omega_{\max }-\frac{t}{t_{\max }}\left(\omega_{\max }-\omega_{\min }\right)
$$

The size of the inertia weight $\omega$ determines how much the particle inherits from the current speed, and a suitable w will help the PSO balance its search capabilities. Formula (2) and (3) become the standard particle swarm formula. For simple description, this paper is called BPSO.

\section{Improved PSO}

The global optimal value in a particle swarm is always superior to the current fitness of all individuals. If $F_{T}$ is the average of the current fitness of all particles, then $F_{T}=\frac{1}{m} \sum_{i=1}^{m} F\left(x_{i}[T]\right), x_{i}[T]$ is the number of particle size $m$ at the position where the particle $i$ is currently iterated $T$, In the process of searching for the minimum value, the , then is defined. In the process of the optimization of the maximum value, the $F\left(p_{g d}[T]\right) \leq F_{T}$, then ${ }_{C=} F\left(p_{s d}[T]\right) / F_{T}$, The synthesis of two cases can be expressed as

$$
C=\frac{\min \left(F\left(p_{g d}[T]\right), F_{T}\right)}{\max \left(F\left(p_{g d}[T]\right), F_{T}\right)}
$$

$C$ is called particle aggregation factor. Obviously, $1<C \leq 1$, which is reflects the current degree of aggregation of all particles, and also reflects the diversity of particles to a certain extent.

In standard particle swarm, particles gather to the best position in their own history, neighborhood or group history, forming the rapid convergence effect of particle population, which is prone to fall into local extremum, premature convergence or stagnation [11]. The main reason for this phenomenon is due to the loss of diversity of particle populations. Therefore, the improved algorithm is proposed, in which the size of aggregation is introduced to measure the diversity of population, and the global extremum $p_{g d}$ is randomly disturbed according to the size of population diversity. The evolutionary stagnation step $t$ is used as the initial condition, and random disturbance is carried out on the individual extremum.

For each particle, the $d$-dimension $(1 \leq d \leq D)$ according to the modified equation is as follows:

$$
\begin{gathered}
\text { if } \quad \text { rand }<C \quad \text { or } \quad t_{i d}>T_{i d} \\
\text { Then } p_{i d}=r_{3} * p_{i d}, p_{g d}=r_{4} * p_{g d} \\
v_{i d}(t+1)=\omega^{*} v_{i d}(t)+c_{1} r_{1}\left(r_{3} * p_{i d}-x_{i d}\right)+c_{2} r_{2}\left(r_{4} * p_{g d}-x_{i d}\right)
\end{gathered}
$$

where: $t_{i d}$ is the number of individual evolutionary stagnation steps; $T_{i d}$ is the individual evolutionary stagnation step threshold; $r_{3}$ and $r_{4}$ are random numbers that follow the $\mathrm{U}(0,1)$ distribution. This improved PSO algorithm is called an IPSO. 


\section{Application of the IPSO-BP Algorithm in Liquid-propellant Rocket Engine Fault Detection}

The working state of the liquid rocket engine is non - linear and can be detected by a method based on nonlinear identification and fuzzy recognition. The backpropagation (BP) neural network is used in the system identification to select an appropriate network model to approximate the actual system. Therefore, the trained network can be used to simulate the operating parameters of the rocket engine, and then the working state of the LRE is detected according to the comparison result.

The general steps of a IPSO-BP algorithm are:

Step 1: Select LRE data samples to determine the neural network input layer neuron number $I$ and output layer neuron number $O$, and determine the hidden layer neuron $M$;

Step 2: Determine the relationship between PSO particles and BP neural network parameters;

Step 3: Calculate the fitness function. Evaluate the fitness of each particle;

Step 4: Determine the best location for each particle found $p_{i d}$, and the best location $p_{g d}$ for the entire group found;

Step 5: Using (8), (2) and the recalculated position of the particle velocity;

Step 6: Assessment of fitness. If the new position of the particle is better than the current $p_{i d}$ value, the value of the update $p_{g d}$ is the best position of the individual particle; if the best position of the group particle is better than $p_{g d}$, the update $p_{g d}$ is the best position for the group particle.

Step 7: Calculate the degree of aggregation of each particle group according to equation (7), and calculate the number of evolutionary stagnation steps of the particle's optimal value. Add the perturbation factor according to equation (9) to disrupt the particle's current search path and increase the probability that the particle will jump out of the local extreme value.

Step 8: If the number of iterations is less than the set maximum value or the error parameter is less than the set error value, skip to Step 3.

Step 9: Pass the resulting optimal individuals to network parameters.

The fault detection of LRE is generally indicated by the change of the parameters of the rocket engine. These faults are mapped out by the change of rocket engine parameters.

\subsection{Data Discretization and Uniformity}

The parameters of the rocket engine value changing with time (especially in the switch machine), the useful information of test data, the continuous time discrete for one moment, according to need to choose different time parameters. In addition, because each test data acquisition is not long inconsistent, and the detection parameters are different, therefore, the need for data sampling the same time, the extraction parameters have been of data, and the data were normalized.

\subsection{Data Selection}

In order to ensure the correctness of the test results, the data with obvious errors are eliminated. According to the statistical experience and law of data mining [12], when the ratio of positive sample (fault state data) and negative sample (normal state data) reaches about 3:7, the model obtained by training can better reflect the distribution law of data. The classification results based on this model will also have high accuracy and confidence. In addition, there are many monitoring parameters; usually, the thermodynamic parameters are selected as the performance indicators of LREs. This work selects representative and weakly related parameters as inputs, for example, the oxygen pump inlet pressure, oxygen pump temperature, hydrogen pump inlet pressure, post-pump temperature and gas generator chamber pressure [13]. 


\subsection{Simulation Results and Analysis}

A certain amount of data from the sample data should be selected as a training sample for the neural network. MATLAB software is used to simulate and compare the BP and SPSO-BP algorithms. The training mean square errors (MSEs) are shown in Fig. 1.

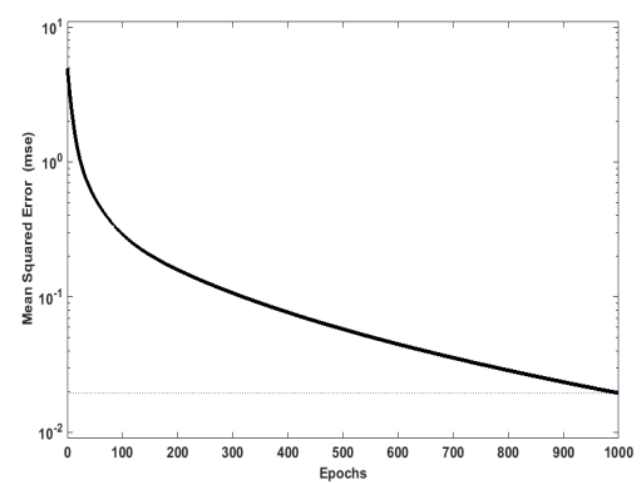

(a)

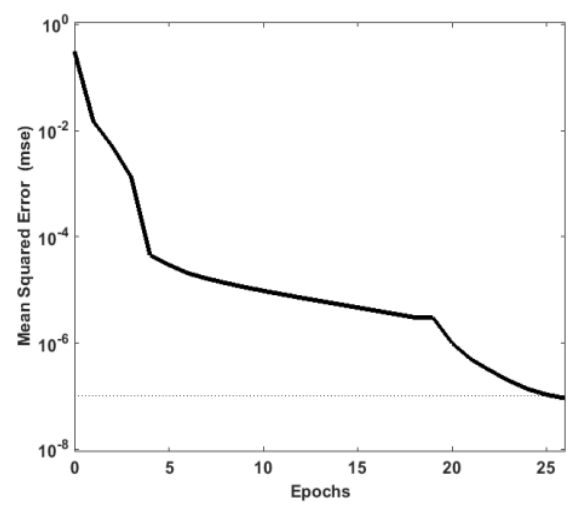

(b)

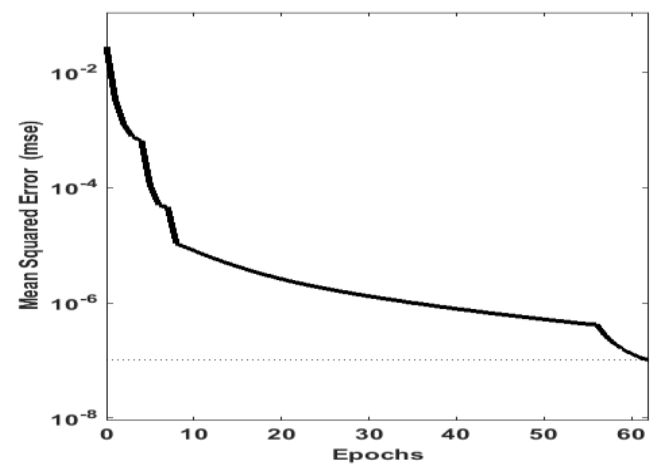

(c)

Fig. 1 Under the same conditions of training parameters and data samples, the MSE curves of BP and SPSO-BP neural network training are compared.

(a)MSE curve of the BP neural network training, (b) MSE curve of the BPSO-BP neural network training

(c) MSE curve of the IPSO-BP neural network training.

The MSE of the BP neural network is in a straight decline at the beginning of training, and the final MSE is far from the set mean square error of 1.0e-7. The MSE of BPSO-BP network after 25 iterations reaches the expected setting. The MSE of IPSO-BP network after 60 iterations reaches the expected setting. It can be seen that the MSE of the IPSO-BP and BPSO-BP algorithm is much smaller than the BP network algorithm under the same initial value and training times. Because the complexity of IPSO algorithm is higher than that BPSO$\mathrm{BP}$, the iterative step size of IPSO-BP training is slightly larger than BPSO-BP algorithm.

\subsection{Diagnosis of Sample Data}

Another two sets of fault sample data and two sets of normal sample data are used as test data [14] to verify the performance of the network. If the results of neural network test are closer to 0, there is no fault in LRE. If the result of neural network test is closer to 1, the LRE will fail. If the result of neural network test approaches 0.5 , it shows that the neural network does not reach the optimal value and can't accurately judge whether there is a fault or not. Its detection sample data is normalized, as in Table 1 and the fault diagnosis results of the neural network are shown in Table 2. 
Table 1. Six Sets of Data as Test Samples

\begin{tabular}{ccccccc}
\hline $\begin{array}{c}\text { Numb } \\
\text { er }\end{array}$ & & \multicolumn{3}{c}{ Sample input } & State \\
\hline $\mathbf{1}$ & 0.5008543000 & 0.4849667000 & 0.523365600 & 0.5024934500 & 0.5070645300 & normal \\
$\mathbf{2}$ & 0.4982934400 & 0.4849223400 & 0.5242545540 & 0.50256435 & 0.50516345 & norml \\
$\mathbf{3}$ & 0.76216498 & 0.7921396498 & 0.7623449800 & 0.7543243498 & 0.541236498 & fault \\
$\mathbf{4}$ & 0.535939633 & 0.5290696432 & 0.5234996412 & 0.518909623 & 0.494739328 & fault \\
\hline
\end{tabular}

Table 2. Sample Data by Comparing the Results of the two Neural Network

\begin{tabular}{ccccc}
\hline $\begin{array}{c}\text { Numb } \\
\text { er }\end{array}$ & BP & BPSO-BP & IPSO-BP & State \\
\hline $\mathbf{1}$ & 0.00825749682307 & 0.00011261365 & 0.000014313470933 & $\begin{array}{c}\text { norm } \\
\text { al }\end{array}$ \\
& & 3 & & norm \\
$\mathbf{2}$ & -0.032746016008572 & 0.00000456517 & 0.000001746244978 & al \\
$\mathbf{3}$ & 0.980759893934929 & 0.99784347344 & 0.999946771575011 & fault \\
$\mathbf{4}$ & 0.978431029119595 & 0.99843242235 & 0.999715802901998 & fault \\
\hline
\end{tabular}

From the simulation results of Table 2 and Fig. 1, it can be seen that conventional BP neural networks fall into local optimum in the process of training, and the accuracy of sample data detection is not as high as that of IPSO-BP neural network and BPSO-BP neural network, IPSO-BP and BPSO-BP neural network are compared, the former can accurately express the fault diagnosis "knowledge" to improve the accuracy of fault diagnosis.

\section{Conclusion}

Using the aggregation degree and the number of particle extremum stagnation steps, the perturbation factor is introduced into the standard particle swarm optimization algorithm. The experimental results show that the improved particle swarm optimization algorithm increases the probability of particle jumping out of the local optimum and enhances the global searching ability. It can converge to the global optimum precisely, and its validity is verified by three classical functions. The IPSO-BP algorithm is used to detect the test data of a typical LRE. The simulation results show that the diagnosis results are closer to the real value and can be applied to the fault detection of LRE. Although the complexity of the new algorithm is slightly higher than that of the standard particle swarm optimization algorithm, the performance of the new algorithm is obviously improved. Therefore, when considered synthetically, the algorithm proposed in this paper will have a good prospect of application.

\section{Acknowledgment}

This work was supported by Big Data Research Foundation of PICC (grant No. 20190418CI000008).

\section{References}

[1] Cai, G. B., Li, J. W., et al. (2011). Liquid-Propellant Rocket Engines. Beijing University of Aeronautics and Astronautics Press.

[2] Zhang, Y. L., Wu, J. J., \& Zhu, H. W. (1993). Liquid-Propellant Rocket Engine Health Monitoring Technology. China, Changsha: National University of Defense Technology Press.

[3] Deng, N. Y., et al. (2007). Fault of Aerospace system and Countermeasures. China Aerospace Press.

[4] Yang, E. F., Zhang, Z. P., et al, (1999). State monitoring system of propulsion system using BP-ART hybrid neural network. Journal of the Propulsion Technology, 6. 
[5] Zhang, W., Zhang, Y. Y., \& Huang, X. X. (2003). Multi-fault diagnosis of turbine pump based on neural network. Journal of the Propulsion Technology, 24, 17-20.

[6] Yu, G. B., Gang, D., \& Lin, L. (2015). Aircraft engine fuel flow prediction using process neural network international. Journal of Control and Automation, 7(3), 53-61.

[7] Kennedy, J. (1997). The particle swarm: Social adaptation of knowledge. Proceedings of the IEEE international Conference on Evolutionary Computation.

[8] Van, D. B. F. (2002). an analysis of particle swarm optimizer. South Africa: University of Pretoria, 78-143.

[9] Ruan, Z. H., Yuan, Y., \& Chen, Q. X. (2016). A new multi-function global particle swarm optimization. Applied Soft Computing, 49, 279-291.

[10] Shi, Y., \& Eberhart, R. C. (1988). A modified particle swarm optimizer. Proceedings of the IEEE In't Conf. of Evolutionary Computation.

[11] Ling, S. H., Lu, H., Leung, F. H. F., et al. (2008). Improved hybrid particle swarm optimized wavelet neural network for modeling the development of fluid dispensing for electronic packaging. IEEE Transactions on Industrial Electronics, 55(9), 3347-3460.

[12] Liang, X. (2006). Data Mining Algorithms and Applications. China, Beijing: Peking University Press.

[13] Xie, T., Chen, H. W., Zhang, Y. L. (2000). Research on multi-fault diagnosis of liquid rocket engine based on set-coverage model. Journal of Astronautics, 21(1), 47-54.

[14] Yang, J. C. (2008). Fault detection and diagnosis of liquid-propellant rocket engine based on genetic algorithm. China, Changshan: National University of Defense Technology Dissertation, 2008.

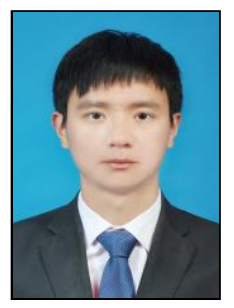

Ningning Li was born in 1993. He was graduated in technology of Tianjin, His current research area is fault detection in liquid-propellant rocket engines. He has been publications published include IEEE Transactions on Aerospace and Electronic, IEEE International Conference on Mechatronics and Automation, International Conference on Information, Electronic and Communication Engineering.

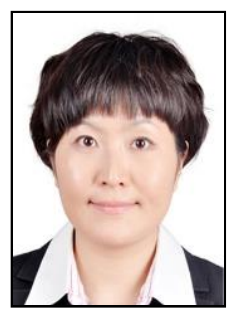

Wei Xue was born in 1981. She was graduated from Xi'an Jiaotong University, and working in the Beijing Aerospace Propulsion Institute, Beijing, China. Her current research area is fault detection. Publications published include IEEE Transactions on Aerospace and Electronic, IEEE International Conference on Mechatronics and Automation, International Conference on Information, Electronic and Communication Engineering.

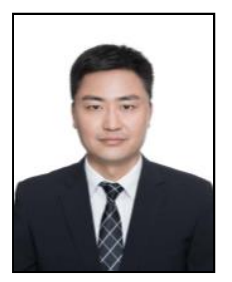

Xiang Guo was born in 1981. He was graduated from Beijing University of Posts and Telecommunications. His current research area is data mining.

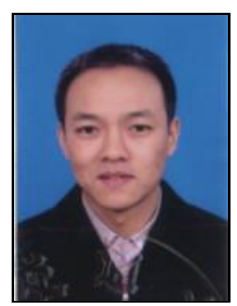

Liang Xu was born in 1980. He was graduated from Xi'an Jiaotong University. His current research area is fault detection and aero-optics. He has been published include IEEE Transactions on Aerospace and Electronic, IEEE International Conference on Mechatronics and Automation, International Conference on Information, Electronic and Communication Engineering. 


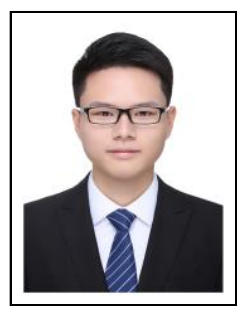

Yuyang Wu was born in 1994. He was graduated in technology of Tianjin. His current research area is fault detection in liquid-propellant rocket engines and Aero-optics.

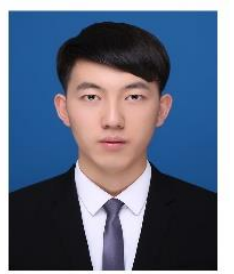

Yao Yuan was born in 1994. He was graduate in technology of Tianjin. His current research area is fault detection in liquid-propellant rocket engines and aero-optics. 